PROCEEDINGS OF THE

AMERICAN MATHEMATICAL SOCIETY

Volume 131, Number 2, Pages 587-592

S 0002-9939(02)06639-X

Article electronically published on July 17, 2002

\title{
MBEKHTA'S SUBSPACES AND A SPECTRAL THEORY OF COMPACT OPERATORS
}

\author{
WEIBANG GONG AND LIBIN WANG
}

(Communicated by Joseph A. Ball)

\begin{abstract}
Let $A$ be an operator on an infinite-dimensional complex Banach space. By means of Mbekhta's subspaces $H_{0}(A)$ and $K(A)$, we give a spectral theory of compact operators. The main results are: Let $A$ be compact. 1. The following assertions are all equivalent: (1) 0 is an isolated point in the spectrum of $A$; (2) $K(A)$ is closed; (3) $K(A)$ is of finite dimension; (4) $K\left(A^{*}\right)$ is closed; (5) $K\left(A^{*}\right)$ is of finite dimension; 2 . sufficient conditions for 0 to be an isolated point in $\sigma(A)$; 3. sufficient and necessary conditions for 0 to be a pole of the resolvent of $A$.
\end{abstract}

\section{TERMINOLOGY AND INTRODUCTION}

Throughout this paper, $X$ will denote an infinite-dimensional complex Banach space and we shall denote the algebra of all bounded linear operators on $X$ by $B(X)$ and the ideal of all compact operators in $B(X)$ by $K(X)$. Let $A \in B(X)$. The nullspace and the range of $A$ will be denoted respectively by $N(A)$ and $R(A)$. $\sigma(A)$ is the spectrum of $A, \rho(A)$ is the resolvent set of $A$ and for each $\lambda \in \rho(A)$, the resolvent of $A(\lambda I-A)^{-1}$ is denoted by $R_{\lambda}(A)$. If $\lambda_{0}$ is an isolated point in $\sigma(A), P_{\lambda_{0}}$ denotes the spectral projection corresponding to $\lambda_{0}$. We say that $A$ is invertible if $A^{-1} \in B(X)$. $A_{M}$ means the restriction of $A$ to an invariant subspace $M$ of $X$ and $A^{*}$ the conjugate of $A$. $\mathbb{C}$ and $\mathbb{N}$ denote respectively the set of complex numbers and the set of positive integers. Finally, $\subseteq$ is used for "is contained in", while $\subset$ is reserved for "is strictly contained in".

The authors of this paper learned the two important subspaces $H_{0}(A)$ and $K(A)$ (see definitions in section 1) from [5]; they were first introduced by Mostafa Mbekhta in [3. In [2, Prop. 49.1], there is an assertion: Let $A \in B(X)$. If $\lambda_{0}$ is an isolated point in $\sigma(A)$, then

$$
R\left(P_{\lambda_{0}}\right)=\left\{x: \lim _{n \rightarrow \infty} \|\left(\left(\lambda_{0} I-A\right)^{n} x \|^{\frac{1}{n}}=0\right\}\right.
$$

or, in the symbols used here,

$$
R\left(P_{\lambda_{0}}\right)=H_{0}\left(\lambda_{0} I-A\right) .
$$

Received by the editors April 19, 2001 and, in revised form, October 2, 2001.

2000 Mathematics Subject Classification. Primary 47A10, 47A11.

Key words and phrases. Spectral theory of compact operators, isolated point of the spectrum, pole of the resolvent operator.

This paper is project 19871048 supported by the NSFC. 
If someone, knowing this fact, had posed the question: what should be the expression for $R\left(P_{\lambda_{0}}\right)$ 's partner $N\left(P_{\lambda_{0}}\right)$ ?, then Mbekhta's subspaces would have been formulated a few years earlier. An already known theorem quoted in [5] as Theorem 3 says: The pole of $R_{\lambda}(A)$ can be characterised by means of the nullspace and the range and for a pole $\lambda_{0}$ of $R_{\lambda}(A)$, both $N\left(P_{\lambda_{0}}\right)$ and $R\left(P_{\lambda_{0}}\right)$ can be expressed in terms of the nullspace and the range of $\left(\lambda_{0} I-A\right)^{\alpha}$, in which $\alpha$ is the order of $\lambda_{0}$. But we didn't see that the nullspace and the range played a similar role for the isolated point in $\sigma(A)$. In [5], $K(A)$ and its partner $H_{0}(A)$ fill the gap. Proposition 4 and Theorem 4 there are remarkable additions to the spectral theory of bounded linear operators.

Motivated by [5], we come to renew a research of the spectrum of the compact operator, the simplest spectrum, in terms of $K(A)$ and $H_{0}(A)$.

\section{Preliminary Results}

Mbekhta's subspaces of $X$ referred to in the title of this paper are: if $A \in B(X)$,

$K(A)=\left\{x \in X:\right.$ there exist $C>0$ and a sequence $\left\{x_{n}\right\}_{n \geq 1} \subseteq X$ such that $A x_{1}=x, A x_{n+1}=x_{n}$ and $\left\|x_{n}\right\| \leq C^{n}\|x\|$ for all $\left.n \in \mathbb{N}\right\}$,

$H_{0}(A)=\left\{x \in X: \lim _{n \rightarrow \infty}\left\|A^{n} x\right\|^{\frac{1}{n}}=0\right\}$.

The following facts are easy to verify and are useful in this paper: for all $n \in \mathbb{N}$,

$$
K(A) \subseteq R\left(A^{n}\right), N\left(A^{n}\right) \subseteq H_{0}(A) ;
$$

for all $\lambda \neq 0$,

$$
N(\lambda I-A) \subseteq K(A)
$$

if $B \in B(X)$ and $A B=B A$, then

$$
H_{0}(A) \subseteq H_{0}(A B) ;
$$

and by this we can prove that $A$ is invertible if and only if $K(A)=X$ and $H_{0}(A)=$ $\{0\}$; finally, [5. Prop. 1] implies that for $A \in B(X)$, if $\lambda \neq 0$,

$$
H_{0}(A) \subseteq R(\lambda I-A) \text {. }
$$

Here in the case that $\lambda_{0} \in \sigma(A)$ is isolated, we have

Theorem 1.1. Let $A \in B(X)$. If $\lambda_{0}$ is an isolated point in $\sigma(A)$, then for all $\lambda \neq \lambda_{0}$,

$$
\{0\} \subset H_{0}\left(\lambda_{0} I-A\right) \subseteq K(\lambda I-A) .
$$

Proof. For all $\lambda \neq \lambda_{0}, \lambda \in \rho\left(A_{R\left(P_{\lambda_{0}}\right)}\right)$, i.e., $(\lambda I-A)_{R\left(P_{\lambda_{0}}\right)}$ is invertible [2, Th. 49.1], and so

$$
(\lambda I-A) R\left(P_{\lambda_{0}}\right)=R\left(P_{\lambda_{0}}\right)
$$

since $R\left(P_{\lambda_{0}}\right)$ is closed, $R\left(P_{\lambda_{0}}\right) \subseteq K(\lambda I-A)$ [5, Prop. 2], while

$$
\{0\} \subset R\left(P_{\lambda_{0}}\right)=H_{0}\left(\lambda_{0} I-A\right) \quad[5, \text { Prop. 4]. }
$$

Theorem 1.1 reveals a few facts that are worth noting. First of all, its proof also tells 
Corollary 1.2. Let $A \in B(X)$. If $\lambda_{0} \neq 0$ is an isolated point in $\sigma(A)$, then

$$
A\left[H_{0}\left(\lambda_{0} I-A\right)\right]=H_{0}\left(\lambda_{0} I-A\right) .
$$

Corollary 1.3. Let $A \in B(X)$. If $\lambda_{0} \in \sigma(A)$ satisfies $K\left(\lambda_{0} I-A\right)=\{0\}$, then $\lambda_{0}$ is the only possible isolated point in $\sigma(A)$.

Proof. If $\lambda^{\prime}$ is an isolated point different from $\lambda_{0}$, we have, by Theorem 1.1,

$$
\{0\} \subset H_{0}\left(\lambda^{\prime} I-A\right) \subseteq K\left(\lambda_{0} I-A\right)
$$

which contradicts the hypothesis.

The following simple necessary condition for $\sigma(A)$ to have more than one isolated point is an immediate consequence of Corollary 1.3.

Corollary 1.4. Let $A \in B(X)$. If $\sigma(A)$ has more than one isolated point, then for all $\lambda \in \mathbb{C},\{0\} \subset K(\lambda I-A)$.

\section{A SPECTRAL THEORY OF COMPACT OPERATORS}

We know that if $A \in K(X)$, then $\sigma(A)$ is countable and has no cluster point except possibly 0; every non-zero number in $\sigma(A)$ is an eigenvalue of $A$ and moreover a pole of $R_{\lambda}(A)$. But little is known about how to characterize 0 to be an isolated point in $\sigma(A)$ with the help of the nullspace and the range of $A$. The following theorem shows that we can do it with $K(A)$, a particular subspace of $R(A)$.

Theorem 2.1. Let $A \in K(X)$. Then the following statements are equivalent:

(1) 0 is an isolated point in $\sigma(A)$;

(2) $K(A)$ is closed;

(3) $K(A)$ is of finite dimension;

(4) $K\left(A^{*}\right)$ is closed;

(5) $K\left(A^{*}\right)$ is of finite dimension.

Proof. Since $\sigma\left(A^{*}\right)=\sigma(A)$, it is easy to see that the equivalence of (1), (2), and (3) implies that of all the statements.

$(1) \Longrightarrow(2)$. See [5, Th. 4].

$(2) \Longrightarrow(3)$. Clearly, since $K(A)$ is closed, $A_{K(A)}$ is compact. Hence, it follows that $A_{K(A)}$ is surjective (4 or [5 Th. 2(a)]) and that $K(A)$ is of finite dimension ([6, Th. V7.4]).

$(3) \Longrightarrow(1)$. Since $A_{K(A)}$ is surjective and $K(A)$ is of finite dimension, $A_{K(A)}$ is invertible. So there exists $\delta>0$ such that $(\lambda I-A)_{K(A)}$ is invertible for $|\lambda|<\delta$.

On the other hand, for $\lambda \neq 0, N(\lambda I-A) \subseteq K(A)$, so we can assert

$$
N(\lambda I-A)=N\left((\lambda I-A)_{K(A)}\right)=\{0\} \quad \text { if } 0<|\lambda|<\delta .
$$

Thus, by the Fredholm Alternative (see [6, p. 334, below]), we have

$$
R(\lambda I-A)=X \quad \text { if } 0<|\lambda|<\delta .
$$

Consequently, $\lambda \in \rho(A)$ if $0<|\lambda|<\delta$. Hence 0 is an isolated point in $\sigma(A)$.

Corollary 2.2. Let $A \in K(X)$. Then:

(1) $\sigma(A)=\{0\}, A$ is quasinilpotent, and $K(A)=\{0\}$ are equivalent;

(2) $\sigma(A)=\left\{0, \lambda_{1}, \lambda_{2}, \cdots, \lambda_{n}\right\}$ if and only if $K(A)$ is closed and $K(A) \neq\{0\}$;

(3) $\sigma(A)=\left\{0, \lambda_{1}, \lambda_{2}, \cdots, \lambda_{n}, \cdots\right\}$ if and only if $K(A)$ is not closed. 
Proof. (1) It is well-known that for $A \in B(X), A$ is quasinilpotent if and only if $\sigma(A)=\{0\}$. If $\sigma(A)=\{0\}$, by [5, Remarque 1.1] we have $H_{0}(A)=X$, and by [5] Prop. 4 or Th. 4] we have $H_{0}(A) \cap K(A)=\{0\}$. Hence $K(A)=\{0\}$. If, on the other hand, $K(A)=\{0\}$, by Corollary $1.3,0$ is the only possible isolated point in $\sigma(A)$. Now, $A$ is compact, so we have $\sigma(A)=\{0\}$.

(2) follows from Theorem 2.1 and (1) above.

(3) follows from Theorem 2.1.

If $A \in K(X)$ and $R(A)$ is closed, then 0 is an isolated point in $\sigma(A)$, since in this case $R(A)$ is finite-dimensional. What can be said about $0 \in \sigma(A)$, if $R(A)$ is not closed? Let us see the example on p. 280 of [6] which throws light on the role of $K(A)$ that cannot be occasionally replaced by $R(A)$.

Example 2.3. Let $X=l^{1}$. Define an operator $A$ on $X$ by

$$
A\left(x_{1}, x_{2}, x_{3}, \cdots\right)=\left(0, x_{1}, \frac{1}{2} x_{2}, \frac{1}{3} x_{3}, \cdots\right) \quad\left(x_{1}, x_{2}, x_{3}, \cdots\right) \in X .
$$

$A$ is a compact quasinilpotent operator. By Corollary 2.2, $K(A)=\{0\}$, so 0 is isolated in $\sigma(A)$. By the definition of $A$, however, $A$ is one-to-one, thus $R(A)$ is not closed.

Proposition 2.4. Let $A \in K(X)$. If there exists $\lambda_{0} \neq 0$ such that

$$
H_{0}\left(\lambda_{0} I-A\right)+H_{0}(A)=X,
$$

then 0 is an isolated point in $\sigma(A)$.

Proof. By [3, Remarque 1.7] and see [6, p. 334, below], for $A \in K(X)$ and $\lambda_{0} \neq 0$, there is $d \geq 1$ such that

$$
K\left(\lambda_{0} I-A\right)=R\left(\left(\lambda_{0} I-A\right)^{d}\right) \quad \text { and } \quad H_{0}\left(\lambda_{0} I-A\right)=N\left(\left(\lambda_{0} I-A\right)^{d}\right) .
$$

By [6, Th. V.7.6], $H_{0}\left(\lambda_{0} I-A\right)$ is of finite dimension. Since $A\left(H_{0}\left(\lambda_{0} I-A\right)\right)=$ $H_{0}\left(\lambda_{0} I-A\right)$ (if $\lambda_{0} \in \sigma(A)$, it follows from Corollary 1.2; if $\lambda_{0} \in \rho(A)$, it is obvious), there exists $\delta>0$ such that

$$
(\lambda I-A) H_{0}\left(\lambda_{0} I-A\right)=H_{0}\left(\lambda_{0} I-A\right) \quad \text { if }|\lambda|<\delta .
$$

Since for all $\lambda \neq 0, H_{0}(A) \subseteq R(\lambda I-A)$, we have

$$
X=H_{0}\left(\lambda_{0} I-A\right)+H_{0}(A) \subseteq R(\lambda I-A) \quad \text { if } 0<|\lambda|<\delta .
$$

By the Fredholm Alternative, $N(\lambda I-A)=\{0\}$. Thus if $0<|\lambda|<\delta, \lambda \in \rho(A)$. Hence 0 is an isolated point in $\sigma(A)$.

Corollary 2.5. Let $A \in K(X)$. If there exists $\lambda_{0} \neq 0$ such that

$$
H_{0}(A)=K\left(\lambda_{0} I-A\right),
$$

then 0 is an isolated point in $\sigma(A)$.

Proof. If $\lambda_{0} \in \rho(A)$, then $K\left(\lambda_{0} I-A\right)=X, H_{0}\left(\lambda_{0} I-A\right)=\{0\}$. If, on the other hand, $\lambda_{0} \in \sigma(A)$, by [5, Th. 4],

$$
X=K\left(\lambda_{0} I-A\right)+H_{0}\left(\lambda_{0} I-A\right) .
$$

Hence for $\lambda_{0} \neq 0$, we have

$$
X=K\left(\lambda_{0} I-A\right)+H_{0}\left(\lambda_{0} I-A\right)=H_{0}(A)+H_{0}\left(\lambda_{0} I-A\right) .
$$

By Proposition 2.4, 0 is an isolated point in $\sigma(A)$. 
The remainder of this paper deals with the pole of the resolvent of $A$.

Theorem 2.6. Let $A \in K(X)$. Then the following statements are equivalent:

(1) $0 \in \sigma(A)$ is a pole of $R_{\lambda}(A)$;

(2) there exists $q \in \mathbb{N}$ such that $\operatorname{dim} R\left(A^{q}\right)<\infty$;

(3) there exists $n \in \mathbb{N}$ such that $K(A)=R\left(A^{n}\right)$;

(4) A has finite descent.

Proof. (1) $\Rightarrow(2)$. By [2, Prop. 50.2] or [6, Th. V.10.1], the descent of $A$ is the order of 0 as a pole of $R_{\lambda}(A)$ and if we denote the order by $q$, we have $R\left(A^{q}\right)=N\left(P_{0}\right)$, where $N\left(P_{0}\right)$ is closed. Hence, $\operatorname{dim} R\left(A^{q}\right)<\infty$, since $A^{q}$ is compact.

$(2) \Rightarrow(3)$. In this case, there exists a finite $n \geq q$ such that

$$
R\left(A^{n+1}\right)=R\left(A^{n}\right) \text {, i.e., } A R\left(A^{n}\right)=R\left(A^{n}\right) .
$$

Since $R\left(A^{n}\right)$ is closed, we have $K(A) \supseteq R\left(A^{n}\right)$; while $K(A) \subseteq R\left(A^{n}\right)$ is always true, we obtain $K(A)=R\left(A^{n}\right)$.

$(3) \Rightarrow(4)$. If $R\left(A^{n}\right)$ takes the place of $K(A)$ in the equality $A K(A)=K(A)$, we have $A R\left(A^{n}\right)=R\left(A^{n}\right)$. This tells us that $A$ has finite descent.

$(4) \Rightarrow(1)$. By $[\underline{6}$, Th. V10.5].

Let $A \in B(X)$. We call the number

$$
\gamma(A)=\inf _{x \in X \backslash N(A)} \frac{\|A x\|}{d(x, N(A))}
$$

the minimum modulus of $A$, where $d(\cdot, \cdot)$ denotes distance (see [1] or [6]).

Proposition 2.7. Let $A \in K(X)$. If

$$
\varlimsup_{n \rightarrow \infty}\left[\gamma\left(A^{n}\right)\right]^{\frac{1}{n}}>0
$$

then 0 is a pole of $R_{\lambda}(A)$ and $\sigma(A) \neq\{0\}$.

Proof. If 0 is not a pole of $R_{\lambda}(A)$, then by Theorem 2.6, for $n \in \mathbb{N}, R\left(A^{n}\right)$ is not closed. Thus, by [6] Th. IV 5.9], we have for $n \in \mathbb{N}, \gamma\left(A^{n}\right)=0$ and so

$$
\varlimsup_{n \rightarrow \infty}\left[\gamma\left(A^{n}\right)\right]^{\frac{1}{n}}=0
$$

which contradicts our assumption.

Now we know that 0 is a pole of $R_{\lambda}(A)$. Denote by $p$ its order; we have by [5, Th. 5] $N\left(A^{p}\right)=H_{0}(A)$. If $\sigma(A)=\{0\}$, then, by Corollary 2.2 and [5, Th. 4] $H_{0}(A)=X$, and so $N\left(A^{p}\right)=X$ i.e., $A^{p}=0$. We again have for $n \geq p, \gamma\left(A^{n}\right)=0$ which yields the same contradiction.

\section{ACKNOWLEDGMENTS}

The authors would like to thank the referee for his/her useful suggestions. Among other things, in particular, the SVEP used in the proofs of the first version were replaced with the Fredholm Alternative, so that the proofs become elementary. 


\section{REFERENCES}

1. C. Apostol, The reduced minimum modulus, Michigan Math. J. 32 (1985), 279-194. MR 87a:47003

2. H. Heuser, Functional Analysis, John Wiley \& Sons, 1982. MR 83m:46001

3. M. Mbekhta, Generalisation de la decomposition de kato aux operateurs paranormaux et spectraux, Glasgow Math. J. 29 (1987), 159-175. MR 88i:47010

4. M. Mbekhta, Sur la theorie spectrale locale et limite des nilpotents, Proc. Amer. Math. Soc. 110 (1990), 621-631. MR 91b:47004

5. C. Schmoeger, On isolated points of the spectrum of a bounded linear operator, Proc. Amer. Math. Soc. 117 (1993), 715-719. MR 93d:47007

6. A. Taylor and D. Lay, Introduction to Functional Analysis, 2nd ed., John Wiley \& Sons, 1980. MR 81b:46001

Department of Mathematics, Qufu Normal University, Qufu, Shandong, 273165, PeoPLE'S REPUBLIC OF CHINA

E-mail address: gongwb@ji-public.sd.cninfo.net

Department of Mathematics, Qufu Normal University, Qufu, Shandong, 273165, PeoPle's RePUblic OF ChinA 